Cahiers d'études italiennes

1 | 2004

NOVECENTO... E DINTORNI

Dire la guerre?

\title{
Guerre et violence
}

Matrices traumatiques de l'écriture d'Erri De Luca

Dominique Vittoz

\section{(2) OpenEdition}

Journals

Édition électronique

URL : http://journals.openedition.org/cei/240

DOI : $10.4000 /$ cei. 240

ISSN : 2260-779X

Éditeur

UGA Éditions/Université Grenoble Alpes

\section{Édition imprimée}

Date de publication : 15 novembre 2004

Pagination : 119-142

ISBN : 978-2-84310-057-4

ISSN : $1770-9571$

\section{Référence électronique}

Dominique Vittoz, «Guerre et violence », Cahiers d'études italiennes [En ligne], 1 | 2004, mis en ligne le 15 mai 2006, consulté le 26 mars 2021. URL : http://journals.openedition.org/cei/240 ; DOI : https:// doi.org/10.4000/cei.240 


\title{
GUERRE ET VIOLENCE
}

\section{MATRICES TRAUMATIQUES DE L'ÉCRITURE D'ERRI DE LUCA}

\author{
Dominique Vittoz \\ Université Jean-Moulin Lyon 3
}

Dans Aceto, arcobaleno, livre essentiel pour la thématique de la guerre et de la violence chez Erri De Luca, le personnage de l'ouvrier ex-terroriste raconte un jour son secret au narrateur dont il est régulièrement l'hôte: il a participé à la lutte armée et perpétré deux homicides. Cette longue journée de révélations restera unique dans leurs échanges et quand le narrateur l'évoque, il en donne l'explication suivante: "Ainsi commença ce jour. Tu parlas, non par besoin d'avouer, mais pour impliquer une autre personne» $(A A, \mathrm{p} .30)^{1}$.

L'écriture chez De Luca n'est ni performance ni épanchement. Écrire a une visée active. Ses livres veulent impliquer, nous impliquer, nous charger d'un poids pas commode, sans pour autant en décharger ni l'auteur ni ses personnages. Au moins autant qu'écrivain engagé, catégorie d'une autre époque, De Luca est un écrivain engageant, au sens où il veut engager celui qui le lit, ne pas le laisser en dehors, mais faire de lui un témoin désormais averti. Ses textes n'ont en revanche rien d'engageant au sens d'avenant, attirant, séduisant ou aguichant. Ses récits campent des personnages sévères, au passé rêche, dominé par les valeurs du travail, de l'engagement dans une cause politique ou une foi religieuse, de la fidélité amoureuse et souvent de la chasteté, du respect filial, de la dignité sans compromission, de la parcimonie matérielle, de la solidarité avec les plus démunis, ou encore, quand il s'agit de personnages d'adolescents, des jeunes gens taciturnes, volontiers solitaires, souvent disgracieux, privés d'insouciance, tourmentés par des responsabilités écrasantes. Dans ses textes non fictionnels, le ton est souvent âpre et sentencieux, la volonté moralisatrice déclarée, les références principalement choisies dans l'univers biblique, les sujets traités jamais bénins, les

1. Pour toutes les citations, nous donnons notre traduction et renvoyons à la pagination du texte original en édition italienne (voir notre bibliographie en fin d'article). Le renvoi aux œuvres de De Luca suit la convention suivante :

AA ; Aceto Arcobalo - APR; Altre prove di risposta - AS; In alto a sinistra - CR; Cattività. Ritratti dal carcere - LC; Lettera da una città bruciata - M; Montedidio - NN; Non ora, non qui - NO; Nocciolo d'oliva - NT; Una nuvola come tappeto - OA ; Opera sull'acqua - OP; Ora prima - PR; Un papavero rosso all'occhiello senza coglierne il fiore - TM; Tu, mio. 


\section{DOMINIQUE VITTOZ}

affirmations et injonctions rarement consensuelles. Enfin ses poésies se partagent entre la méditation biblique, déjà présente dans ses traductions et dans deux recueils de variations en prose inspirées de l'Ancien et du Nouveau Testament (NT et NO), et le bilan moral d'une vie et d'un siècle, dont le poème Tessera donne une formulation exemplaire: «Beaucoup d'amis en prison et en exil/paient pour moi aussi le vingtième siècle» $(O A$, p. 114). Ces deux vers nous semblent être une bonne porte pour entrer dans l'œuvre de De Luca quand on entend en explorer les racines traumatiques, là où se nouent conscience et écriture.

\section{Repérages: De Luca un "auteur en chair et en os"}

Erri De Luca connaît la notoriété grâce à ses récits, part la plus connue de son œuvre où fiction, écriture d'essai, poésie, traduction s'encastrent solidement, jamais étrangères les unes aux autres.

Cette construction remarquablement solidaire revendique pour fondations une série d'expériences vécues et méditées, essentiellement de nature politique et historique. Cette indissociabilité de l'œuvre et de l'auteur fonde le pacte de réception, éveillant chez le lecteur, comme le remarque Attilio Scuderi "une curiosité extrême envers l'auteur, son autobiographisme, le court-circuit entre dimension narrative et biographie ${ }^{2} »$. Il est vrai qu'en France comme en Italie, à chaque nouvelle publication, la presse décrit et souligne le trajet existentiel fort de l'auteur, sa personnalité hors normes dont on connaît les étapes marquantes: la rupture en 1968, à dix-huit ans, d'avec une famille aisée, les années de militantisme à l'extrême gauche, puis la condition d'ouvrier, simple manœuvre, l'étude solitaire et profane de l'hébreu, l'engagement sur le terrain pendant la guerre en ex-Yougoslavie, et jusqu'à ses hardiesses de grimpeur et d'alpiniste. Battage médiatique et commercial, pourra-t-on dire, mais ce n'est pas que cela.

Dans L'ombra lunga dell'autore. Indagine su una figura cancellata, Carla Benedetti nous a appris à reconsidérer avec plus de réalisme et de bienveillance ce qu'elle nomme la fonction-auteur, son omniprésence que la postmodernité a voulu refouler ${ }^{3}$. Et la formule d'«auteur en chair et en os» qu'elle utilisait pour Pasolini dans Pasolini contro Calvino nous semble bien adaptée à De Luca qui parait paisiblement imperméable aux phobies postmodernes, dont la mort de

2. A. Scuderi, Erri De Luca, Fiesole, Cadmo, 2002, p. 104. Cette monographie est précieuse comme première tentative de présentation d'ensemble du travail de De Luca. Elle ne peut souvent qu'amorcer des pistes d'interprétation, en particulier autour des notions de spreco et de tuttaltro dont nous reparlerons plus avant. Elle met opportunément l'accent sur la dimension autographique de l'écriture de De Luca mais sans en tirer toutes les conséquences en particulier sur la figure de l'auteur. En revanche, l'analyse stylistique est solide et stimulante et propose un jugement de valeur assez tranché sur les trois derniers romans $(T u$, mio, Tre cavalli et Montedidio). Enfin le long entretien qui clôt le livre constitue une mise à jour utile des positions politiques, culturelles, religieuses et littéraires de De Luca telles qu'il souhaite les expliciter.

3. C. Benedetti, L'ombra lunga dell'autore. Indagine su una figura cancellata, Milan, Feltrinelli, 1999, en particulier le chapitre $1: \vec{E}$ morto l'autore? p. 9-32. 
l'auteur fut la plus voyante, et étranger par conséquent aux paralysies dénoncées par Carla Benedetti. De Luca n'est pas de ceux qui, pour reprendre les termes de Carla Benedetti, écrivent une «littérature totalement épuisée, embaumée dans sa géométrie inerte et ayant désormais accepté en toute mélancolie, tel un destin déjà écrit, sa propre impuissance à avoir une incidence sur le monde ${ }^{4}{ }$. Et s' $s^{\prime}$ il en est ainsi, c'est peut-être parce qu'il n'est pas en conflit avec sa "fonction-auteur", les raisons de l'écriture faisant chez lui tout un avec les choix de vie.

Ce qui caractérise l'écriture de De Luca, c'est la position d'autorité où elle se situe d'emblée aussi bien pour l'écrivain que pour son lecteur. Ses textes de fiction, ses traductions, ses poésies, comme ses interventions de nature journalistique ont en commun d'affirmer sans détours un système de valeurs morales où se conjuguent une fascination pour la loi hébraïque, un ascétisme personnel, l'honnêteté et la fidélité envers un passé de luttes et d'espoirs collectifs, une sévérité fin de siècle, un sentiment et une pratique de la fraternité. De Luca n'a aucune difficulté à qualifier lui-même ses interventions de "petits sermons " $(P R$, p. 127). Cette ambition éthique est justifiée par le sentiment que l'utopie, placée au ras des choses, reste un horizon possible de nos actions.

\section{Une écriture agissante}

Du côté du lecteur, son œuvre qui s'est imposée d'abord par ses qualités stylistiques, s'est rapidement augmentée de l'aura supplémentaire que peuvent conférer l'authenticité, le témoignage, l'expérience vécue et le charisme. Le lecteur de De Luca sait désormais combien ses textes de fiction, ainsi que ses réflexions portant sur les années de la contestation, sur le monde du travail et de l'émigration, sur la Naples des tremblements de terre, sur la guerre en ex-Yougoslavie, sont le fait d'un acteur directement impliqué.

Du côté de l'écrivain, le propos est net dès les origines: l'écriture est un corps à corps avec le monde. Écrire ou être écrit, affirmait Erri, le signataire des six Lettere a Francesca ${ }^{5}$ publiées en 1990, tel est l'enjeu pour l'individu face à un monde ni pacifique ni pacifié. L'enjeu politique de l'écriture, bien réel et jamais évacué, est le fruit d'un matérialisme de grande ampleur.

En effet pour De Luca, la tension écrire-être écrit est originelle, elle est le fondement du rapport de l'être humain au monde qui l'entoure, entendu cette fois au sens de monde créé, de matrice matérielle. Dans un beau texte de 1996, intitulé Materia scritta et publié dans le recueil Alzaia, De Luca retrace en un tableau épuré les étapes constitutives de son acte d'écrire: d'abord sa journée de travail sur le chantier qui laisse ses mains blanchies de poussière de marbre, puis son retour à la maison, son dîner composé d'une seiche dont l'encre sur ses mains

4. Idem, Pasolini contro Calvino. Per una letteratura impura, Turin, Bollati Boringhieri, 1998, p. 197.

5. Nous citons tous les titres des ouvrages de De Luca dans leur version originale par cohérence car, pour deux d'entre eux, le titre choisi pour l'édition française efface des connotations dont nous aurons besoin plus avant; il s'agit de Non ora, non qui (Une fois, un jour) et de Ora prima (Première heure). 


\section{DOMINIQUe VitToZ}

dépose des traces noires, son regard sur ses mains marquées de signes noirs sur fond blanc, semblables à un alphabet et, pour finir, ses mains ainsi écrites qui se mettent à écrire à leur tour "sur un bout de table pas encore débarrassée» (p. 64). Ici les conditions d'émergence de l'écriture sont claires: "Si je peux faire des pages en écrivain, c'est parce que moi-même ce soir je suis écrit au noir de seiche et à la poussière de marbre, sur dos et paume de mains. [...] j'écris la matière qui m’a écrit» (p. 64).

Cette circulation fluide des choses vers les mots, sans barrage, sans filtre, remonte le courant originel, des noms vers les choses, quand Adam nommait la création en lisant les noms à même la matière. Ce n'est pas la vision platonicienne où les noms forgés par le nomothète saisissent quelque parcelle de l'être. Les noms sont donnés à l'humanité au même titre que la création et font bloc avec le réel. Le matérialisme de De Luca manie de l'absolu.

Enfin sur un troisième plan, celui de la construction de récits, le même binôme écrire-être écrit fonctionne. Dans Altre prove di risposta, De Luca se défend d'être un "vrai écrivain" pour se présenter ainsi:

pour quelqu'un à moitié hébété et à moitié muet, écrire des histoires, c’est souvent se sentir écrit par une histoire, en provenir. La mettre par écrit ne peut pas me donner le titre d'auteur, mais celui plus adapté de rédacteur. (p. 26)

La figure du rédacteur ancre sans équivoque l'écriture de fiction à une matrice historique. Sa source est l'expérience vécue, la sienne propre ou celle d'autrui. L'expérience des hommes est la matière première de l'Histoire, mais la relater ne signifie pas la répéter. Le rédacteur n'est ni passif ni transparent, et encore moins résigné:

je reviens sur le passé avec le désir de lui donner une autre possibilité, de faire advenir quelque chose qui aurait pu se passer à l'époque, mais qui n'eut pas lieu pour un cheveu, ou qui n'eut pas lieu exactement de cette façon-là ou pas avec cette promptitude de réflexes, avec cette intelligence du cœur qu'on n'a pas quand les choses arrivent, qu'on ne peut avoir qu'après, en faisant advenir le passé une deuxième fois 6 .

Nous avons jusqu'ici cité des réflexions de l'écrivain sur sa pratique, formulations hors texte de ses intentions pour le texte. Mais dans ses textes de fiction aussi, par exemple dans la nouvelle In alto a sinistra, on trouve l'expression - et presque mot pour mot - de ce programme d'écriture.

Implication existentielle, inscription dans une filiation et une voie, conscience aiguë des erreurs et des horreurs du passé, ont chacune leur incidence sur ce travail de réécriture du monde. La littérature est «la deuxième chance donnée au monde ${ }^{7}$ " car les mots restent lointainement porteurs d'une force originelle dont la Bible est le lieu premier et inimitable. Comme la critique le souligne avec plus ou moins de pertinence, la fréquentation assidue, prolongée et totale des textes bibliques (lecture, commentaire, traduction) marque d'une empreinte décisive l'écriture de De Luca ${ }^{8}$.

6. E. de Luca, in «La scrittura come occasione", intervista di Grazia Casagrande, in Caffe lettarario di Alice. it, 15 octobre 1999.

7. Idem, in Dialogo con Erri De Luca, in A. Scuderi, Erri De Luca, cit., p. 138.

8. Voici deux évaluations opposées du poids de la Bible dans l'œuvre d'Erri De Luca. Qualifiant 


\section{Une écriture lestée}

De Luca rappelle souvent que le terme hébreu qui signifie «mot», davàr, signifie

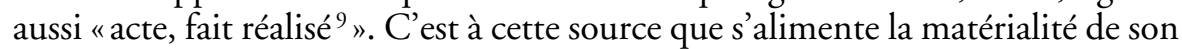
écriture, où l'action est centrale. Ses récits concernent des faits et gestes, ses personnages sont avant tout ce qu'ils font et l'adolescent de Montedidio en est l'exemple le plus évident, un peu trop appuyé peut-être, dans le parallèle entre son entraînement musculaire au lancer du boomerang et son mûrissement affectif et moral. Il y a toujours déjà présence des actes dans les mots, l'écriture et le monde ne sont pas à distance mais constitutivement emmêlés et c'est au cœur de cette mêlée que De Luca se place.

Écrire est réaliser la compénétration entre sa propre voix et celle du monde, en acceptant que les vents la bousculent. Dans le texte Voci al vento de Un papavero rosso all'occhiello senza coglierne il fiore, De Luca se démarque des «vrais écrivains» qui d'après lui,

défendent leur mélodie des canailleries de la nature. Je ne me sentais pas de cette espèce-là, je n'aurais jamais le geste d'exclure le vent du micro, ni la vie incohérente et sans-gêne des pages que je remplissais. (p. 78-79)

Cette écriture qui se défend du monde est pour de Luca une parole plus mutilée que celle qui s'en laisse traverser et même bousculer ou malmener.

Le vent dans son ouvre occupe une grande place. Il fait partie des quelques valeurs répertoriées dans la poésie Valore, laquelle constitue une dense profession de foi existentielle: "Je considère comme une valeur de savoir dans une pièce où est le nord, quel est le nom du vent qui sèche le linge " $(O A$, p. 98). Dans Aceto, arcobaleno, le vent règne en toute-puissance, maitre à bord dans la maison du narrateur à son agonie, destinée à s'écrouler à la fin du livre, quand la voix même du narrateur n'est plus qu'un "souffle au-dessus des décombres» (p. 117), d'où tout peut alors recommencer, souffle de l'écriture.

La place importante qu'a le vent dans la représentation de l'écriture est bien sûr d'ascendance biblique. Traduisant et commentant Qohélet, De Luca met au centre de sa lecture de ce livre amer et cinglant un vent à la fois littéraire et sacré. Les paroles de K. s'y mesurent et comme lui, l'écrivain se trouve simultanément empli de ce souffle du monde et par celui-ci dépossédé. Mais le résultat n'est pas égal à zéro, il y a un reste et, pour De Luca, le texte est ce reste, ce résidu, inestimable dépôt après que tout a été gaspillé.

l'écriture de De Luca de «consapevole senza essere mimetica del testo-matrice che contiene tutti gli altri, cioè, la Bibbia", Myriam Swennen Ruthenberg articule toute sa lecture de Tu, mio comme une recherche ontologique entre eau et terre, selon la dialectique régissant les rapports entre consonnes et voyelles en hébreu (voir "Eccomi sulla spiaggia-confine di Tu, mio: Erri De Luca, il mare e l'asciutto", in Narrativa, Université Paris X, juin 2001, n 20-21, p. 169-180). En revanche, Lucio Izzo semble n'accorder à l'univers biblique qu'une place de résultante et non de source, encore moins de moteur de l'écriture: «il tema dell'esilio, un tema connaturato alla cultura dell'ebraismo diasporico [...] costituisce il ponte, la mediazione culturale attraverso la quale De Luca si avvicina alla dimensione biblica.» («La patria napoletana di Erri De Luca", in Italies, Université d'Aix-en-Provence, 2002, nº 6, vol. 2, p. 681692). Le plus souvent la critique n'évoque qu'incidemment cet intérêt de De Luca pour la Bible.

9. Voir le chapitre L'età "davarista ", in PR, p. 86-87. 


\section{Ce qui est inutilisable n'est pas vain}

C'est par cette notion de gaspillage (spreco) que De Luca se risque à traduire le mot clé du livre de Qohélet, l'hébreu hèvel que Jérôme a traduit par vanitas et que la dernière en date des traductions françaises aussi rend par vanité ${ }^{10}$. Ses arguments philologiques sont convaincants qui relient le mot hèvel au nom propre Abel, le premier assassiné, le premier sang gaspillé d'un homme qui n'aura pas de descendance et dont le nom, contrairement à ceux d'Adam, d'Ève, de Caïn ou de Set, ne donne lieu dans les Écritures à aucune élucidation, poussant donc l'exégète à en proposer une.

L'assassinat d'Abel est le premier gaspillage et le mouvement du monde en produira un immense, succession de violences et de morts. L'humanité est alors ce qui a réchappé, le reste d'un gaspillage. Mais il ne s'agit pas seulement d'une vision abstraite de la condition humaine. Ce que De Luca lit dans Qohélet, c'est la condition historique de survivants dans laquelle ont été jetés des hommes. La condition juive d'après la Shoah, telle qu'il l'évoque dans $T u$, mio et Montedidio est entièrement placée sous ce signe. Dans In alto a sinistra, le père souligne que "ce qui reste ne démontre rien et ne remplace rien de ce qui a été perdu" ( $A S$, p. 126). Le personnage formule cette affirmation à propos des pages lues au cours de son existence, mais elle possède une portée beaucoup plus large.

Le reste est en même temps ce qui a une valeur absolue, celle de la survie, et aucune valeur de justification ni de rachat. Le gaspillage est à la fois total et producteur de résidu. Là aussi, c'est une expérience historique qu'il traduit en ces termes, celle de sa génération engagée dans les luttes sociales et politiques des années soixante-dix et dont certains de ses acteurs, les militants encore emprisonnés, connaissent cette condition de "reste d'une époque âpre et achevée [...], dépôt séché qu'une nation civilisée ne sait pas gratter du fond de la casserole " (PR, p. 33), «reste de XXe siècle mis aux fers» (CR, p. 23). Et il s'agit pour De Luca d'une condition existentielle historique dont il se sent solidaire, par-delà ce qui a séparé sa route de celle des terroristes.

Pour De Luca, le monde est marqué au double sceau du gaspillage et du résidu, et son écriture en répète la dialectique. C’est dans cette perspective que nous comprenons son choix des formes brèves, ainsi que la densité de son style, toujours très contenu : extrême rareté des suffixes dans une langue, l'italien, où ils abondent, absence parfois déconcertante de l'article défini, adjectivation sous haute surveillance, centralité des verbes, prudence et retenue syntaxiques, choix lexicaux forts et massifs comme autant de rocs. L'écriture selon De Luca rejoint la sculpture selon Michel-Ange, cet «art d'ôter » que l'artiste distinguait de «l'art de déposer" qu'est la peinture. L'écriture contient toutes les éliminations auxquelles il convient de savoir se résoudre. Elle se soumet à un processus de distillation. De Luca s'interdit l'épanchement, la consolation : «Si un soir tu écris parce

10. Voir Paroles de Qohélet, traduction de Marie Borel, Jacques Roubaud et Jean L'Hour, in La bible, Paris-Montréal, Bayard-Médiaspaul, 2001, p. 1632-1666. 
que tu te sens malade, si tu écris pour te procurer un soulagement, alors après, efface tout» (APR, p. 39). Ainsi se dessine une conception du monde et de l'écriture où l'individu n'a sa marge de liberté et de sens que dans ce qu'il lui est donné de sauver, le peu qu'il lui est donné de sauver de la submersion et de la coercition. Ce paradigme qui ordonne l'univers de De Luca traduit deux réalités irréductibles: la guerre, comme héritage et horizon, et les contraintes physiques et morales du travail manuel salarié le plus subalterne.

Pour l'ouvrier autodidacte que fut longtemps De Luca, l'étude de l'hébreu et de la Bible restait circonscrite à cette "heure avant", pour reprendre une des significations du titre Ora prima, c'est-à-dire cette heure avant le moment du départ pour le chantier, heure qu'il soustrayait au sommeil afin de "saisir quelque chose de chaque nouvelle journée avant que la fatigue ne la [lui] vole» (p.5). De Luca se réfere alors, en l'interprétant de façon positive, à l'image employée par le prophète Amos, le premier à annoncer de sévères châtiments à Israël: tout comme le berger ne sauve de la gueule du lion que quelques lambeaux du corps de sa brebis, ainsi Dieu ne sauvera des juifs de Samarie que «un pied de lit et le bout d'une couverture ${ }^{11}$ ". Dans l'interprétation qu'en donne De Luca, c'est à ces «restes inutilisables» qu'est confié le salut. (OP, p. 5) Et il en va de même pour ces restes de l'Histoire que sont les rescapés, et pour ses textes, restes des mots éparpillés.

Ce qui est inutilisable n'est toutefois pas vain et le refus de De Luca de suivre la tradition de la traduction de hèvel par vanité le prouve. Certes la littérature ne compense rien, comme l'adolescent de $T u$, mio en acquiert la certitude: «Caia a subi des choses qu'aucun récit ne peut équilibrer» (p. 82). Mais l'écriture constitue bien un troisième pôle entre le silence et le geste. Car d'un côté, le silence est ce dont, malgré leurs penchants mutiques, bégaiements, enrouements, mues et autres défauts de voix, les personnages de De Luca savent qu'ils doivent sortir pour que ne s'écroule pas ce que "aucun récit ne soutient plus", selon la formulation de Aceto arcobaleno que nous avons déjà relevée. Et de l'autre côté, le geste, l'acte, la violence sont en dernier ressort une impasse puisqu'ils ne peuvent pas "corriger le passé» comme l'affirme l'adolescent de Tu, mio à l'instant même où il vient d'accomplir son geste de vengeance, dans la phrase qui clôt le roman. Entre la vacuité du mutisme, du silence "qui se fige comme du sang versé au milieu des gens" qu'évoque l'ouvrier d'Aceto arcobaleno (p.42) et l'acte justicier de l'adolescent de Montedidio "pour lequel il n'y a pas de place sur le rouleau ${ }^{12}$ ", l'Histoire garde sa sourde et nue violence. C'est ce que l'écriture de De Luca souhaite affronter.

\section{Les pieds sur terre}

Chez De Luca, la fiction n'est pas une fin, et à peine un moyen. Dans Altre prove di risposta, il l'affirme nettement, son travail ne concerne pas des personnages,

11. Amos, 3, 12, traduit par Valère Novarina et Marc Dubreucq, in La Bible, cit., p. 1061.

12. Ce sont les derniers mots de Montedidio. 


\section{DOMINIQUe VITTOZ}

mais des personnes: «Je ne possède pas de personnages, je ne les écris pas, je ne les invente pas; j'écris des personnes, c'est-à-dire un morceau d'humanité qui a déjà eu lieu, qui a déjà été créé et constitué" p. 27). Pour lui, se mêler à l'humanité, en partager les peines comme il l'a fait pendant la guerre en ex-Yougoslavie, est nécessaire, mais non suffisant. Acteur de convois humanitaires pour la Bosnie en compagnie de bénévoles catholiques, il est frappé par la foi en Dieu que professent ses compagnons d'expédition. Au regard de leurs actions qu'il perçoit comme lestées d'une "étrange épaisseur» (p.93), il sent les siennes, les mêmes pourtant, sans ancrage. Et c'est alors l'écriture qui lui permet de faire le poids.

La littérature ainsi conçue se pratique au ras du sol, à hauteur de rez-dechaussée, de trottoir, de sol, de terre, de roche, là où s'appuient les pieds, volontiers déchaussés, qui sont la partie du corps humain la plus présente dans les textes de De Luca: les pieds déformés du narrateur de Non ora, non qui (p. 7577), les plantes de pieds robustes et calleuses de l'ami missionnaire dans Aceto, arcobaleno (p.77), les pieds de l'adolescent de $T u$, mio nus pour la pêche et pour la vengeance (p.11,107, 113), les pieds disparus de l'épouse argentine victime de la junte militaire puis les pieds amoureux de Laila et du jardinier dans Tre cavalli (p. 78 et 68-69), les pieds sans chaussures des gamins napolitains pour qui le cordonnier juif de Montedidio fabrique généreusement le nécessaire (p.18). Et, pour quitter les textes de fiction, ce sont aussi les pieds d'Ovidio Bompressi à qui il rend visite en prison. Aux pieds, qui sont "la partie la plus prisonnière d'un corps emprisonné", De Luca consacre un très beau texte, Elogio dei piedi. (APR, p. 77). Et la toute première raison d'hommage qu'il énonce est le fait «qu'ils sont loin de la tête», manière d'affirmer à l'égard de cette dernière une méfiance qu'on peut sans doute entendre aussi comme une défiance envers ce que serait une attitude intellectuelle pure, coupée de la réalité et de son contact direct. L'indissociabilité entre les écrits et les actes charpente les textes de De Luca et le choix systématique pour ses récits de personnages aux métiers manuels est l'écume visible de cette structure profonde.

C'est du contact direct avec les choses que le personnage de l'ouvrier dans Aceto, arcobaleno tire son intelligence du monde, comme le relève le narrateur intrigué qui l'écoute formuler une réflexion sur la douleur physique à partir de la façon dont un clou, en se fichant dans le bois, vient y occuper une place non arbitraire: "Tes idées m'étonnaient. "Ce ne sont pas de vraies idées mais des impressions, des choses trouvées sous les sens» » (p.30). Les sens, et non l'esprit, sont le canal par lequel l'homme et le monde se comprennent, au sens littéral de s'interpénétrer.

Un des premiers livres de De Luca, est un recueil de cinq nouvelles intitulé $I$ colpi dei sensi, une nouvelle pour chacun des sens ${ }^{13}$. Il s'agit de cinq épisodes

13. Peu après la rédaction de notre étude, ces cinq nouvelles ont été rééditées dans un recueil plus vaste, intitulé Il contrario di uno (Feltrinelli, 2003). 
écrits à la première personne auxquels une brève introduction donne un statut autobiographique. Les sens y sont les acteurs, non comme source de plaisirs, éventuellement coupables (on entend en écho dans le titre les colpe dei sensi, les "fautes des sens»), mais comme détonateurs essentiels de la connaissance, comme piliers d'un "ce que je sais" de son siècle, que De Luca concentre en quelques précipités irréductibles. Sauf pour le dernier épisode qui transcrit une expérience strictement intime, les réalités qui viennent le frapper de plein fouet (émigration, guerre, exterminations nazies, univers carcéral) relèvent de nœuds historiques qui inlassablement donnent matière et corps à ses textes, qu'ils soient de fiction ou de réflexion.

\section{Les coups de l'Histoire}

Les thèmes récurrents posés comme essentiels par I colpi dei sensi correspondent à des moments tragiques de l'Histoire, où des hommes sont écrasés injustement: les émigrants d'hier et d'aujourd'hui sacrifiés aux déséquilibres de la mondialisation; les populations civiles devenues, en guerre, depuis la deuxième mondiale, des cibles massives; les juifs exterminés par millions; les anciens militants des années de plomb encore emprisonnés. Ces vaincus, ces "opprimés» de l'Histoire, pour reprendre le terme qu'utilise Walter Benjamin, se donnent la main par-dessus les époques et les générations. De Luca reçoit avec toute leur force ce que Horkheimer appelait les "protestations qui viennent du passé ${ }^{14}$ », et c'est la raison de leur réitération presque obsessionnelle dans ses romans, dès le premier, Non ora, non qui, où le thème du mal apparait d'une façon marginale, mais en rien anodine, puisque l'enfant écoutant sa mère évoquer injustices ou malheurs, s'en considère rien moins que responsable. ( $N N$, p. 58). Ensuite, tous ses romans vont largement donner place aux «coups de l'Histoire».

Dans Aceto arcobaleno, les vers d'Ossip Mandelstam placés en exergue situent de prime abord l'ambition du récit: "Siècle mien, brute mienne, qui saura/Plonger les yeux dans tes prunelles/Et ressouder avec son sang/Les vertèbres des deux siècles? ${ }^{15}$ ". Les trois vies dont le narrateur mourant répète les récits, ont rencontré la rébellion, la lutte armée, la prison, la pauvreté du tiers monde; elles sont un condensé d'espoirs, de combats et de souffrances que la dernière phrase du livre évoque en termes de décombres balayés par le dernier souffle du narrateur, dans une vision très proche de la célèbre thèse IX que Benjamin consacre à l'ange de l'histoire ${ }^{16}$.

14. M. Horkheimer, Kritische Theorie, Francfort, S. Fischer, 1968, cité in Michaël Lowy, Walter Benjamin: avertissement d'incendie. Une lecture des thèses "Sur le concept d'histoire», Paris, Puf, 2001, p. 37.

15. O. Mandelstam, Le siècle, in Tristia et autres poèmes, choisis et traduits du russe par François Kérel, Paris, Gallimard, 1975 et 1982, p. 111.

16. «La cataratta dei lampi si è placata, io sono un soffio sopra le macerie.», E. De Luca, $A A$, p. 117. 


\section{DOMINIQUe VITTOZ}

Dans $T u$, mio, la thématique se concentre sur l'extermination des juifs par les nazis et la deuxième guerre mondiale. La curiosité obstinée du jeune narrateur sur ces sujets se heurte aux réticences des adultes, qu'il peut vaincre seulement au prix de ne pas leur dévoiler de quelle exigence de vérité aiguë elle se nourrit.

Récit d'un meurtre tout à fait privé (celui du souteneur de Laila qui ne peut reprendre sa liberté qu'à ce prix), Tre cavalli est empli d'échos de guerres: la première guerre mondiale; la guerre en Croatie, énième erreur d'appréciation des premiers concernés, comme en témoigne un vieil homme, croate; la résistance armée contre le junte militaire argentine; la guerre des Falklands; l'oppression du peuple kurde.

Enfin Montedidio entrelace deux destinées, celle d'un cordonnier juif d'Europe centrale, Rafaniello, rescapé mais bloqué à Naples après guerre dans son voyage pour la Palestine, et celle d'un adolescent napolitain dont la révolte croît en même temps que sa force. Quand, dans la scène finale, le jeune garçon a poussé dans le vide l'agresseur de Maria, le cri qui lui sort de la gorge, «braiement d'âne qui [lui] arrache les poumons» (p. 142) est le même braiement d'âne qui, pendant sa descente en Italie, avait déchiré les oreilles de Rafaniello pourtant "pleines de guerre et de ses pires bruits» et avait fait pleurer ses yeux qui "pendant toute la guerre» étaient restés secs (p. 117). Le jeune narrateur est lui-même chargé de la mémoire de la guerre, à l'échelle de sa famille comme à celle de la ville, à travers les récits de la mort de son oncle tué dans un bombardement (p. 72) et des actions de maître Errico et du concierge don Ciccio, qui participèrent avec les habitants du quartier à la résistance contre les Allemands (p. 82-83 et 115).

Ces réitérations ne sont pas le fait d'une crise d'inspiration, mais plutôt le résultat d'un inlassable commentaire des mêmes faits. On retrouve là une attitude qui est celle requise pour la fréquentation de la Bible, où il s'agit de lire ce qui de nombreuses fois déjà a été lu, et de l'expliquer à nouveau, une fois encore. De Luca revient obsessionnellement sur les mêmes thèmes, dans ses textes d'essai et dans ses textes de fiction, comme hanté.

Les voix du passé, des "ancêtres", de "ceux qui ne [1]'ont pas connu» (APR, p. 9) parce que venus avant lui, ne parviennent pas à l'écrivain dans la confortable torpeur des rêves, même éveillés. Elles n'apportent pas de consolation. Elles ne sont pas l'occasion d'effets esthétiques ou de raffinements intellectuels. Ces voix sont la présence concrète et exigeante, encore qu'hallucinatoire, de l'humanité qui nous a précédés. Dans Montedidio elles prennent même corps ou du moins forme, d'esprits, de fantômes. C'était déjà le cas dans Tufo où l'enfant narrateur contemple un immeuble déserté et hanté, métaphore pour De Luca du XX siècle: "Tandis qu'expire le siècle auquel j'appartiens tout entier, j'y pense comme à un immeuble vide où personne ne veut plus habiter. Seuls résistent les absents, cramponnés à des pièces qui n'existent plus» (p. 30). À contre-courant des oublis consolateurs, ou pire manipulateurs, cet enfant-là restera pénétré des traces de mémoire de son siècle.

En revanche, les jeunes de la génération suivante apparaissent à De Luca allégés de tout lest historique. Dans un texte de Pianoterra, il les décrit flottant à la 
surface de l'eau, «indifférents aux grands fonds, à l'abysse qui, en contre-poussée, soutient leur légèreté» (p. 17). Et cette confortable situation de flottement est selon lui périlleuse: «Parfois je pense qu'ils voyagent mieux que moi, d'autres fois je pense qu'ils vont au hasard, comme le plancton dans la gueule de la baleine» (p. 17).

La première empreinte qui va marquer De Luca, est celle de la deuxième guerre mondiale dont - rappelons-le -, né en 1950, il n'est pas le contemporain. Dès lors la guerre (celle-ci et d'autres postérieures), cette violence de groupe exercée à des fins politiques et légitimée peu ou prou, sera omniprésente dans ses écrits où elle apparaît comme la réalité dominante du champ politique. Par cette focalisation, l'après-guerre à Naples tel que De Luca l'assimile et le représente est bien différent de celui de son concitoyen Raffaele La Capria, auteur que nous évoquons ici car il est un des rares écrivains italiens que De Luca cite nommément, quand il évoque son parcours de lectures formatrices. ( $L C$, p. 34) Ce que De Luca attribue à La Capria, c'est la transmission du monde de l'avant-guerre comme s'il considérait que le roman majeur de son aîné, Ferito a morte (1961), cette plongée hébétée dans le continuum historique anesthésiant où Naples s'englue à partir de la fin des hostilités, indifférente aux secousses de l'Histoire, n'était pas recevable comme représentation de la ville après septembre $1943{ }^{17}$. Nous en voyons la raison dans la façon dont les personnages de La Capria ont digéré les années de guerre: "On commence à parler de guerre et on finit en parlant de femmes", constate un de ces vitelloni parthénopéens ${ }^{18}$. Une telle position est radicalement étrangère à la vision de De Luca et en cela $T u$, mio nous semble être aussi une entreprise de réfutation de la parole des aînés, de certains aînés. En effet, les représentations littéraires que De Luca désigne en revanche comme "l'arbre de transmission père-fils» sont les livres de «Levi, Cassola, Pratolini, Fenoglio, Pavese poète» (LC, p. 33-34). Constatons que ce sont tous des auteurs chez qui l'expérience de la guerre nourrit des œuvres qui atteignent une dimension épique. C'est une indication précieuse pour interpréter une œuvre dont Filippo La Porta reconnaît qu'il ne réussit pas à repérer les «ascendances littéraires ${ }^{19}$ ".

\section{La question de la violence}

Il y a dans l'univers de De Luca une matrice traumatique qui ne relève ni de l'affect ni de l'inconscient, mais qui au contraire est clairement formulée et touche au champ politique et citoyen. En ce point névralgique, se mêlent l'héri-

17. Dans Ferito a morte, Naples est «la Foresta Vergine», où une génération «si è messa fuori della Storia ", R. La Capria, Ferito a morte, Milan, Mondadori, 1984, p. 116 et 46.

18. Ibidem, p. 45

19. F. La Porta, La nuova narrativa italiana. Travestimenti e stili di fine secolo, Turin, Bollati Boringhieri, 1995, p.79. La nouvelle édition augmentée (1999) ne présente pas de complément ou d'ajout au paragraphe consacré à De Luca. 


\section{DOMINIQUe VitToz}

tage historique encore brûlant de la deuxième guerre mondiale et l'expérience de la révolte collective des années soixante-dix, avec son horizon de combats, y compris violents et armés.

Dans Aceto, arcobaleno, le roman où on voit bien, quinze ans plus tard, que De Luca avait déjà planté sans hésitations tous les jalons de son écriture à venir, le personnage manifestement le plus important, l'ouvrier devenu terroriste ${ }^{20}$, explique sa relation à la violence:

Enfant, je voyais des violences partout. Je prenais dans les étagères de mon père, des livres sur la guerre et je grandissais en sentant que je devais répondre de blessures, d'offenses qu'une autre génération avait fait retomber sur celles à venir. Le vingtième siècle était devenu un siècle antique, plein de malédictions. Quand je vis dans les rues les coups, les affrontements, j'étais déjà prêt. Dans le premier pavé que j'ai lancé était déjà contenu le fait de tuer et d'être tué. La politique fut pour moi l'organisation d'une colère, un cal qui s'épaississait. Passèrent non pas des semaines, mais des années, j'ai connu les armes, j'ai tué des hommes, un et puis un autre. (p.32)

Voici un schéma explicatif - et pas justificatif - remarquablement net et tranché qui relie solidement le recours à la violence des militants de gauche, dans les manifestations de rue et dans les luttes sociales et jusqu'au choix minoritaire de ceux qui devinrent terroristes, à l'explosion de violences sans précédents que fut la deuxième guerre mondiale.

Dans un texte postérieur, n'appartenant pas au registre de la fiction, De Luca redonne à cette analyse des contours spécifiquement autobiographiques et plus personnels que dans Aceto, arcobaleno. Dans une série de trois lettres à Angelo Bolaffi publiés dans MicroMega en 1998, se livrant à un examen rétrospectif à trente ans de distance, de l'année 1968, il commence par évoquer, dans des termes très proches de ceux qu'il a employés pour le personnage d'Aceto, arcobaleno, sa prise de conscience de ce que fut la deuxième guerre mondiale et le poids de responsabilités dont il héritait. Mais il continue en soulignant la spécificité de cette «éducation sentimentale» qui fut la sienne:

Je ne sais pas si quelque chose de semblable t'est arrivé, à toi, ou à d'autres jeunes de notre âge.

[...] Il ne manquait pas à Naples, en Italie, dans le monde, à la moitié des années soixante, d'injustices et d'abus de pouvoir tout frais. Mais ils ne me prenaient pas au collet aussi violemment que ceux qui avaient eu lieu vingt ans plus tôt. Ces ouvrages sur la guerre m'avaient isolé.

Le grand nombre, la foule, je l'ai rencontrée ailleurs et seulement parce qu'elle était si vaste et encombrante qu'elle m'entraînait avec elle. ( $L C$, p. 34-35)

Dans cet isolement au sein de la foule, que le texte de fiction n'explicitait pas avec autant de netteté, se trouve peut-être une clé pour comprendre la stature toute particulière de De Luca.

De Luca n'entend pas mettre en regard l'expérience de la résistance armée des pères et le choix de la "critique des armes" que, à l'intérieur du mouvement contestataire, firent plusieurs centaines de jeunes de la génération suivante. Pour De Luca, leur violence fut une «contre-force non autorisée » qu'il définit comme

20. Des trois épisodes, c'est celui de l'ami ouvrier qui est de loin le plus long, puisqu'il occupe la moitié du livre. 
une "nécessité épuisante et douloureuse de pratiquer des exemples de résistance» (p. 94 et 96). Et il prend soin de préciser la portée de ce terme: «Je n’emploie pas ce mot pour me réclamer de La Résistance avec des majuscules, qui dura peu et fut la mise en pratique du principe de légitime défense» (p. 96). Il n'y a donc pas là de continuité combattante.

Son propos est en revanche de souligner ce que fut sa condition inconfortable d'adolescent découvrant dans les livres et dans les récits des adultes les horreurs d'une Histoire dont les programmes scolaires ne disaient encore rien: «les récits des adultes me poussèrent à chercher leur passé et me transmirent la responsabilité d'en être le fils et la suite» ( $P$., p. 16). Cette transmission orale, de personne à personne, génère un sentiment de responsabilité face à la violence et au mal, que De Luca introduira régulièrement dans ses fictions. Dans tous ses livres, les personnages sont confrontés à la violence, et l'exercent.

En cherchant les modulations du leitmotiv de la violence, on remarquera que sa première apparition revêt la forme du silence: en effet, dans son premier roman, Non ora non qui, il n'y a pas de problématique de la violence. On ne trouve que trois allusions à la guerre, qu'on peut qualifier de marginales et qui concernent uniquement les retombées économiques des bombardements et des restrictions sur la vie familiale. Et pourtant deux indices nous poussent à relier ce roman aussi à la veine encore souterraine où se tressent traumatisme de la guerre et révolte des années soixante-dix. Dans une lettre ouverte à Ovidio Bompressi, publiée dans MicroMega en 1996, où - nous y reviendrons - il soutient la thèse de la responsabilité collective de la gauche révolutionnaire italienne dans l'assassinat du commissaire Calabresi, De Luca emploie la même expression non ora non qui. Rappelons que dans le roman, c'est la mère qui l'emploie pour réprimander ses enfants ${ }^{21}$. Dans l'article, elle est rapportée comme négation de l'espoir et de la pratique communistes que vivait alors une partie importante de la génération de De Luca:

Quel genre d'hommes sommes-nous devenus tous deux, Ovidio? Rien d'autre que les mêmes, mais sans la communauté du communisme. Nous l'avons vu ce mot, c'était facile de le faire, nous n'avons pas cru ceux qui disaient de lui : «Pas maintenant, pas ici». (LC, p. 17)

Dans le roman aussi, le narrateur apporte son démenti à cette injonction paralysante, mais sans virulence. Sa désobéissance est involontaire, puisque c'est de sa mort qu'il s'agit:

Je ne peux pas t'obéir, je n'en ai plus le temps. Ça va se passer juste maintenant, et dans cet étrange endroit. "Pas maintenant, pas ici». Tu avais raison, beaucoup des choses qui me sont arrivées furent des erreurs de temps et de lieu, des choses à vous faire dire: pas maintenant, pas ici. Toutefois contre cette vitre de bus, je m'aperçois que je suis en un moment et en un lieu qui m'étaient réservés depuis longtemps. ( $N N$, p. 43-44)

Dans les deux cas d'emploi de cette expression fortement inhibitrice, c'est en fin de compte le sentiment d'adéquation, d'opportunité, d'à propos, qui prend le

21. «Mi guardi con il cruccio severo dove resta quel tuo eterno rimprovero rivolto a noi bambini: non ora, non qui. ", $N N$, p. 43. 


\section{DOMINIQUe VitToz}

dessus, annule l'interdit et contre la résignation. Et là, il nous semble difficile de ne pas rapprocher ce non ora, non qui du titre Se non ora, quando? que Primo Levi donne à son grand roman de la résistance. Cette expression est d'ailleurs celle qu'utilise De Luca quand il évoque l'attente du messie pour les croyants ${ }^{22}$. Il ne faut alors peut-être pas voir dans le récit Non ora, non qui, le seul dans l'œuvre de De Luca à porter la marque dominante de la figure maternelle, uniquement un intime roman des adieux qui, fil à fil, cautérise les liens tranchés entre mère et fils. Mais il convient de comprendre que, par ce silencieux rappel à l'écrivain italien dont l'œuvre s'inscrit le plus dramatiquement dans l'horreur de la deuxième guerre mondiale, le premier livre de De Luca porte lui aussi, comme une résonance en attente, le bruit et la violence de son siècle. Puis, dans tous les livres suivants, la violence sera un thème central.

Dans Aceto, arcobaleno, l'expérience des armes et du meurtre est le fait d'un militant politique italien entré dans la clandestinité et la lutte armée des années soixante-dix. Quand il décrit ses actes (le meurtre d'un "ennemi» politique, puis celui d'un ancien terroriste devenu dangereux pour l'organisation), il utilise le vocabulaire de la guerre, se définissant comme soldat: «À cette époque-là, j'étais soldat volontaire d'une force que nous promettions provisoire» (p.33). On retrouvera le même vocabulaire dans Tre cavalli, du moins pour la première partie de l'histoire du jardinier, quand il entre dans la résistance armée argentine: le mot guerre revient avec une fréquence particulièrement élevée et, malgré la référence spécifique à la résistance armée contre la dictature, avec une valeur pour ainsi dire absolue, qui permet d'y lire conjointement d'autres références historiques. Voici comment le jardinier explique à Laila la raison pour laquelle il a longtemps vécu en Argentine: "Elle sourit: et pourquoi là-bas? Guerre. Laquelle? Une guerre, il y en a toujours une» (p.34). Voici comment il lui décrit son entrée dans la résistance: "Je suis ici depuis des années pour aimer une femme et maintenant je suis en guerre» (p.47). Et voici comment il répond à l'amie de Mimmo qui lui demande s'il pense avoir été utile: "On est dans une guerre aussi par honte de rester à l'extérieur. Et puis un deuil vous saisit et vous fait rester soldat par colère» (p.62). Il s'agit plus d'une condition existentielle, réduite à ses traits fondamentaux, à des invariants, que d'une expérience contingente liée à un lieu et une époque donnés. La raison nous paraît la suivante: malgré la lettre du scénario, De Luca ne parle pas tant de la résistance en Argentine que de la lutte armée en Italie, glissement de lecture facilité par le fait que l'auteur attribue au jardinier un passé de militant politique au sein de "l'infanterie ouvrière» qui fit de Turin "une ville de pions insurgés contre le reste de l'échiquier» (p. 16). On comprend mieux alors que le narrateur parle de son engagement armé en Argentine non pas en termes de haine personnelle (qui aurait été parfaitement vraisemblable chez l'époux d'une desaparecida) ou de choix idéologique. Bien sûr, c'est d'abord l'homme à qui on a arraché Dvora, qui va prendre les armes:

22. "Questa promessa scritta nei libri sacri e sempre sospesa, ha aiutato le persone di fede a vivere meglio, a dar peso ai gesti, alle parole e a chiedere infine sul limite del tempo loro assegnato : se non ora, quando?», P, p. 57. 
de la maison de nos noces je n'emporte qu'une seule chose de Dvora, ses tennis aux lacets encore noués parce qu'elle les enlève en poussant sur le talon. C'est mon devoir d'en défaire les nœuds et de les tenir prêtes pour elle. C'est ce que j'emporte, hébété par le malheur, en dette pour avoir négligé de prendre soin [...]. (p.78)

Mais c'est la notion de dette qui l'y pousse, c'est-à-dire qu'il est confronté aux mêmes termes de dette que nous trouvons chez les autres personnages de De Luca, ceux qui portent en eux l'exigence de répondre activement des horreurs de la deuxième guerre mondiale.

Il y a un impératif d'action face à l'abus et à l'injustice (ici les arrestations arbitraires des opposants qui débouchaient sur des disparitions par milliers), un devoir de non réserve, dont l'aboutissement est de ne plus se sentir en dette. Le poids de la dette est quelque chose qui lie, attache, entrave, comme le dit Selim reprochant amicalement au jardinier de ne pas lui permettre de payer en vin ou en argent sa dette pour les fleurs qu'il lui fournit gratuitement et dont lui, fait commerce: "Tu ne veux pas de mon argent, tu ne veux pas de mon vin. Comme ça tu lies, tu ne dénoues pas» (p. 100).

Dénouer les lacets, dénouer la dette de l'ami, c'est dans les deux cas la violence et le meurtre qui en sont la voie. C'est avec une image inattendue de noud défait, de liens dénoués, que le jardinier évoque sa pratique de "guerre vagabonde» (p.78), de lutte contre la dictature militaire: «des jours sans lendemain [...], on va déliés à l'affrontement, on va face aux coups sans plier» (p. 86). Et c'est pour "dénouer» ce qui était resté lié que Selim se charge, sans en rien dire, d'accomplir le meurtre du proxénète de Laila, pour "racheter une dette», disproportion incompréhensible si on en restait à la lettre du texte, mais qui s'éclaire quand on entend le mot dette dans une comptabilité plus générale entre bien et mal (p. 107). Ce à quoi nous pousse un autre passage, de Tu, mio cette fois, où s'adressant à son père, l'adolescent employait le mot dette à un double niveau, celui financier et banal de somme d'argent due et celui, moral et exigeant, de torts à réparer (p. 108).

Tre cavalli rapproche deux situations de meurtre, l'un de nature collective et guerrière (les actions armées du narrateur contre des policiers, puis un colonel, argentins), l'autre totalement privé (le meurtre du proxénète par Selim). Mais dans les deux cas, il s'agit d'un poids à prendre sur soi. C'est ce dont Selim a délesté le jardinier: «Un autre homme est à ma place d'assassin sans m'en ôter la faute, il n'ôte que le geste.» (p. 108). Et inversement quand il abandonne la résistance armée, le narrateur en embarquant pour les Falklands sent que «sans armes, [il] ne pèse plus rien» (p.48).

Mais cette légèreté n'est pas de l'inconséquence ou du cynisme. À la place de l'arme, le narrateur désormais porte sur lui un livre. C'est sur cette substitution que se clôt le roman: "Je mets le livre dans la poche intérieure de ma veste, je le cale sur ma poitrine par-dedans. À l'ancienne place de l'arme, maintenant il y a le tout autre» (p. 109). On retrouve dans cette notion de "tout autre» la fonction que, nous l'avons vu, De Luca attribue à la littérature: être une deuxième chance pour le monde, l'occasion d'une correction du passé. Dans le finale de Tre cavalli, la ronde des objets échangés place le livre au rang d'instrument concret et 


\section{DOMINIQUE VITTOZ}

donne au "tout autre» une substance physique. Passé et présent se rejoignent car l'espace de liberté que constitue la radicale différence contenue dans le «tout autre» renvoie autant à un univers inventé prenant forme dans la réécriture «en mieux" du passé, et dont les livres sont les porteurs bénéfiques, qu'à une réalité vécue dont nous trouvons la clé dans un autre texte, non fictionnel, l'article Ognuno di noi poteva, publié trois ans avant Tre cavalli: "Qui peut venir nous dire que le communisme a été prise du pouvoir, police secrète, dictature, goulag: nous n'avons rien été de tout cela et beaucoup de tout autre, à ce moment-là et à cet endroit-là, ouvertement» ( $L C$, p. 17). Ce "tout autre» fut donc une condition existentielle réelle, une utopie qui avait trouvé son topos et son chronos, soulignés avec insistance dans la modulation libertaire allora e lì, «à ce moment-là et à cet endroit-là", qui réfute, encore une fois, le non ora, non qui, "pas maintenant, pas ici», autoritaire dont nous avons déjà vu que De Luca prend le contrepied.

De Luca assigne au jardinier un destin ouvert: à l'avant-dernière page du roman, c'est son deuxième cheval qui meurt et il en faut trois pour faire un homme, comme dit la comptine qui donne son titre au livre.

En revanche, il avait tracé jusqu'au bout le destin du militant d'Aceto, arcobaleno. Après son abandon de la lutte armée, celui-ci rencontre deux réalités, le travail ouvrier et la foi en Dieu. Ni l'une ni l'autre n'ont la capacité de donner une autre inflexion, un autre sens à son existence. La foi religieuse lui reste extérieure et la nostalgie qui l'effleure à cette occasion est d'être, pour sa part, exilé de l'exigence droite de maintenir «les promesses faites quand on est jeune» (p. 68), fidélité possible en revanche pour les hommes de foi tel que le jésuite qu'il rencontre au sanatorium. Quant à son travail de manœuvre, il s'y coule en ne mobilisant que l'application du corps et en refusant toute valeur symbolique ajoutée à cette activité, et plus largement, à sa vie sociale (p.63). Il se déclare extérieur, et cette condition d'extériorité est directement proportionnelle à l'intensité du sentiment antérieur d'appartenance collective qui l'habitait et qui désormais est privé de ses fondements. Le resserrement de champ sur l'unité minimale de l'individu, c'est-à-dire son corps, est alors total. Les dernières paroles de ce personnage concernent son rapport à son corps, qu'il a traité comme une "bête sous le joug", et le bilan de son existence semble alors vouloir se cantonner à une trajectoire physiologique:

[Mon corps] m'a opposé, à moi qui le fatiguais, une patience aveugle, se pliant à tous les jours que je lui ai donnés. Je ne peux pas pénétrer au-delà de son écorce, tout effort le transforme et le conserve à distance. Je tressaille parfois quand je sens la mort qui besogne à l'intérieur, mais c'est un sursaut de l'esprit. À tout moment le corps est prêt à se défaire, chaque jour il est prêt à accueillir la vie et la mort de la même main. (p. 64)

Cette absence de soi préludera au choix de la mort volontaire, du moins accueillie, selon l'annonce qu'en fait l'ami lui-même au narrateur:

«Une nuit d'hiver où il fera très froid, je resterai dormir dehors. Ce sera quand je n'aurai plus la force de gagner ma journée. Ce sera bientôt ». Un matin de cet hiver-là, je trouvai l'étang gelé. (p. 64) 
Bien servie par la sobriété du finale - c'est en effet sur ces phrases que se clôt l'épisode de l'ouvrier -, cette dissolution de l'être, réduit à équilibre algébrique entre ses jours et ses nuits, ne vient toutefois pas effacer complètement ses dernières paroles d'identité, glissées peu avant, presque incidemment, comme une conclusion plutôt inattendue de sa méditation sur son corps: "Je n'ai pas honte de la façon dont je l'ai traité, ni de ce que j'ai fait» (p.64). C'est, au tout dernier moment, le corps habité d'une volonté qui reprend la place, pour une revendication nue de ses actes. Toutes considérations précédentes dûment enregistrées (rappelons les incises récurrentes du narrateur qui décrivent comment les paroles de ses visiteurs pénètrent les murs mêmes de la maison), le reste net de cette existence est une affirmation. Malgré tout, et malgré la violence et le meurtre par lesquels elle dut passer.

Les deux romans Aceto, arcobaleno et Tre cavalli sont reliés par les similitudes de destin des deux personnages de militants politiques devenus combattants. Les deux autres romans, $T u$, mio et Montedidio, constituent eux aussi une espèce de diptyque. Ils sont tous deux situés à une époque antérieure: un moment peu déterminé des années cinquante pour le premier, et l'année 1960 très précisément pour le deuxième. Ils sont tous deux centrés sur le parcours initiatique de deux adolescents de Naples qui se mesurent à la violence et au meurtre à travers leur rencontre avec des personnages juifs d'Europe centrale: la jeune Caia, enfant d'une famille exterminée, et Rafaniello, survivant obstiné et solitaire de tout un village.

Dans $T u$, mio, ce n'est pas seulement l'amour pour Caia qui pousse le jeune narrateur à la venger, c'est une vague puissante de "colère devant un mal qu' [il] ne connaît pas, dont [il] ne sait que quelques noms» (p.74). L'acte de violence (l'incendie peut-être meurtrier de la pension de vacances) est un acte pour l'acte, une réponse, lancée au vent, à la démission et à l'impuissance devant le mal, en l'occurrence les massacres et les déportations dont Nicola le pêcheur a fait l'expérience terrible et dont il transmet le récit à l'adolescent (p. 39-40).

Contre le modèle de son oncle, qui s'est tenu à l'écart de tout combat, et de son père, qui reste étranger à l'indignation de son fils devant l'assujettissement de Naples à la présence militaire américaine, l'adolescent ressent avec une extrême acuité que si «ailleurs la guerre est finie depuis longtemps, chez nous elle continue» (p. 108). À la fin de Tu, mio, dans le dialogue entre père et fils où les deux personnages prennent la mesure des changements profonds qui sont survenus chez l'adolescent pendant cet été crucial, le jeune garçon formule ainsi cette montée d'action qu'il perçoit en lui: «je ne sais répondre à rien, mais mon corps est en train de se charger de l'élan de répondre» (p. 58). Ce sont des termes qu'on retrouvera chez son homologue de Montedidio. En effet, ce ressort musculaire du corps dans $T u$, mio, réapparaît dans le geste répété du lancer de boomerang de Montedidio. Bien que le boomerang soit défini dès le début du livre comme «une arme» (p.9) et que l'adolescent pressente qu'il peut viser et frapper quelqu'un (p.39 et 56), sa trajectoire finalement lâchée n'a pas de cible, mais ouvre une voie dans le ciel, d'abord à l'envol de Rafaniello, le juif rescapé que ses ailes d'ange pourront enfin porter jusqu'à Jérusalem, puis à la chute violente du propriétaire, 


\section{DOMINIQUE VITTOZ}

poussé par l'adolescent. Ce geste meurtrier répond au mal subi par Maria longtemps victime des agressions du propriétaire de l'immeuble, tolérées par ses parents, qui compensent ainsi le non paiement de leurs loyers. Le geste violent contre le suborneur signifie ne pas faire comme les adultes, ne pas «rester regarder le spectacle» comme "Dieu le père qui a vu et n'a pas aidé». Il marque le refus d'encourir ce même reproche que, dans une proclamation presque blasphématoire, la jeune fille qui n'a pas été épargnée, avait adressé au "père éternel» (p. 50). Enfin ce geste de poussée vers le bas trace une trajectoire de violence justicière qui a été précédée, introduite, peut-être autorisée, par une trajectoire inversement symétrique, vers le haut. L'ombre malfaisante qui agresse Maria à la dernière page du livre est poussée à toutes forces vers le bas, à terre, comme peu auparavant le boomerang et Rafaniello avaient tous deux été propulsés vers le haut, dans le ciel, bénéficiant l'un et l'autre d'une poussée, dans les deux cas, pas ordinaire. En effet, pour ce lancer unique, le boomerang reçoit une "poussée jamais obtenue» (p. 141) jusque-là. Quant à Rafaniello, ce sont carrément les esprits qui secondent son décollage en se mettant à "pousser eux aussi par-dessous». Et les deux envols sont salués par une «dernière poussée d'adieu » de l'adolescent levant haut ses bras contre le ciel. Ces poussées nécessaires contiennent la violence des coups, et c'est ce mot que l'adolescent avait employé quelques pages plus tôt pour les désigner (p. 138).

Maria aussi relie la force de vie avec une certaine violence quand, à la même page, elle compare les battements de cœur de son jeune fiancé à un gamin qui "lance des pierres contre un mur» (p. 138). De Luca lui attribue une image qui, ramenée à un contexte plus proche de nous, peut facilement en évoquer une autre, nettement plus offensive, de lancers de pavés ou autres cocktails Molotov. Ce télescopage interprétatif pourra paraître abusif. Pourtant, ce lancer tant retenu, en suspens depuis les premières pages du livre, sans cesse répété à vide et finalement destiné en toute utopie à atteindre une étoile et non à frapper un agresseur, nous semble constituer un arrêt sur image, possible en littérature, impossible dans la réalité, une icône, l'indécidable justification de la violence chez un auteur pour qui elle fut une réalité:

Je me rends compte que pour ceux qui lisent mes récits, mes personnes sont des personnages, et que par conséquent la violence prend une valeur de passage littéraire... En revanche pour moi, elle n'est que ce qui a eu lieu ${ }^{23}$.

Et cette réalité vécue a la formulation la plus concise et la plus nue:

à la question si nous étions violents, je réponds: oui. Je ne rappelle pas en préambule que telle était l'époque, et le lieu, le sang déjà versé et les injustices. Je réponds: oui. ( $L C$, p. 76)

Voilà quelque chose d'incompressible, qui leste existence et écriture.

Dans les deux romans, le finale se répète: l'acte de violence, dénouement de la tension dont tout le livre a construit le crescendo, porte sa propre malédiction, exprimée par la phrase négative qui le qualifie et clôt l'œuvre: «derrière moi

23. Dialogo con Erri De Luca, in A. Scuderi, cit, p. 138. 
explosait un feu qui ne pouvait pas corriger le passé » (TM, p. 114) et «il n'y a pas de place pour mon cri sur le rouleau ni au-dessus de Montedidio" ( $M, \mathrm{p} .142)$. La contradiction reste ouverte entre le recours à la violence qui s'impose peu à peu, aussi inéluctable que le mûrissement progressif de chacun des adolescents, et ce qui, irrémédiablement, reste en elle d'inapproprié (dans le cas de $T u, m i o$ ) ou d'inacceptable (pour Montedidio). On ne peut s'empêcher de lire dans ces conclusions un regard porté sur la violence non légitimée - contrairement à celle de la guerre qui, à tort ou à raison, l'est ontologiquement - à laquelle recoururent les militants de la gauche révolutionnaire italienne et dont l'exacerbation engendra la lutte armée. Chef du service d'ordre de Lotta Continua, De Luca fut pendant des années aux prises avec la violence, face à la police, contre les militants d'extrême droite, dans les piquets de grève. Il distingue cette violence-là de la lutte armée, sur la base d'un présupposé qui ne relève pas de l'éthique, mais de la politique:

En ce qui me concerne, la critique fondamentale à l'égard des terroristes, la chose que je leur reprochais et que je leur reproche est l'idée que la lutte armée fût, en soi, la seule forme possible de lutte politique ${ }^{24}$.

C'est sur cette base qu'apparaît la cohérence de sa position dans le débat sur la digestion historique de ces années chaudes que la société italienne se doit désormais de réaliser. En effet, dans cet après-terrorisme où l'Italie est entrée depuis presque vingt ans, De Luca refuse de se démarquer de ceux qui firent le choix des armes, et dont il ne fut pas. $(P$, p. 27) Loin de regretter, renier ou se repentir d'actes subversifs, De Luca se proclame citoyen toujours en marge dans un pays qui n’a pas encore donné de conclusion politique aux années de plomb. $(P, \mathrm{p} .28-$ 29). Il n'est donc pas question pour De Luca de se targuer d'une innocuité confortable et à cet égard, sa position dans l'affaire Calabresi est exemplaire. Il n'a de cesse depuis les rebondissements judiciaires provoqués par les aveux de Leonardo Marino et les condamnations en particulier d'Adriano Sofri et Ovidio Bompressi, de rappeler à quel point en ces années-là, la violence était le fait, non pas de quelques esprits isolés dangereusement échauffés, mais de tout un mouvement dans sa globalité. Pour De Luca, ce qui garde un véritable sens, en amont des issues inconfortables qui attendaient ces militants, tous choix confondus, c'est l'identité collective, le communisme existentiel, qu'il partagea avec beaucoup d'autres jeunes Italiens et qui prenait la forme du pronom sujet "nous»: "C'est un nous qui prenait parti, et une bonne partie d'une génération" ( $P$, p. 27). Quand il évoque l'accès à la parole politique que lui a permis cette expérience générationnelle, il emploie des termes proches de ceux que, auteur de Montedidio, il utilise pour décrire la conséquence physiologique de son geste meurtrier chez l'adolescent narrateur. ( $M$, p. 142) La même image d'obturation et de libération unit l'adolescent de Montedidio au jeune homme des années soixante-dix, et met en regard leur expérience de la violence, suggérant peut-être que si le cri du premier n'a pas trouvé de place, celui du deuxième avait, en

24. Ibidem p. 129. 


\section{DOMINIQUe VitToZ}

revanche, trouvé la sienne. Cet élan collectif, cette «jeunesse publique de rue» ( $L C$, p. 172) où chacun trouvait place et sens, fut, selon De Luca «la dernière fois que le pronom "nous» eut une consistance nationale, affranchie de tout sigle d'entreprise ou de parti» ( $P$, p. 27). Et quelques années plus tard, il affirme que non seulement une telle identité collective ne s'est plus reformée ensuite, mais qu'elle n'avait même jamais existé auparavant: "Ça s'est passé alors, et jamais plus, et jamais auparavant». ( $L C$, p. 35) Et en effet, les deux adolescents de $T u$, mio et de Montedidio sont seuls dans leur violence et c'est aussi pour cette raison que leur acte, unique, ne trouve pas de place, et reste sans avenir.

Si les deux romans les plus ancrés dans la période de la deuxième guerre mondiale semblent indissociables de l'expérience et de la réflexion de De Luca sur la violence des années soixante-dix et quatre-vingt, inversement sa réflexion sur l'après-terrorisme renvoie à son tour en miroir à la deuxième guerre mondiale. En effet, quand De Luca imagine que, grâce à une amnistie et à la libération des anciens terroristes encore emprisonnés, la page des années de plomb est enfin complètement tournée, la référence qui vient sous sa plume pour évoquer cette démobilisation libératrice et légitime, est un titre de film, celui du «beau film de Comencini», Tutti a casa (La grande pagaille), c'est-à-dire un film sur la deuxième guerre mondiale. $(P$, p. 29) Cette circulation entre les deux périodes historiques laisse ouvert le problème dérangeant du recours à la violence, y compris armée. N'oublions pas en effet que le film de Comencini a pour conclusion le choix par les anciens soldats en débandade d'empoigner les armes en résistants. Évoquer ce film dans un texte qui envisage la libération des ex-terroristes et le solde de tout compte de deux décennies témoigne bien du lien indissoluble chez de Luca entre guerre et violence politique autre.

\section{Conclusion}

De Luca est un écrivain hanté par la guerre, cette "épreuve de privations et de terreurs" que, selon lui, la littérature serait en devoir de représenter. C'est du moins ce qu'on entend dans sa condamnation du Moravia auteur des Indifférents au moment où toute une jeunesse sacrifiée pendant le premier conflit mondial restait hors champ de la littérature. ( $P R$, p. 119) Le reproche est un peu anachronique si on considère que Moravia, né en 1907, n'a pas partagé la condition des jeunes soldats de 1915-1918. Mais qu'importe aux yeux de De Luca: écrivant dix ans après cette guerre, Moravia aurait dû en ressentir tout le drame, un peu comme De Luca écrivant quatre décennies après la deuxième guerre mondiale s'est senti directement touché par ce déchaînement de violence et le gaspillage des morts. Donner voix à ceux à qui une mort injuste l'a arrachée, et plus largement à ceux qui ne peuvent pas ou plus la faire entendre, relève pour De Luca d'un véritable devoir: "J'ai eu le don d'être écouté par écrit, j'essaie de l'honorer en écrivant sur ceux d'entre nous qui ont arrêté de parler" ( $L C$, p. 18). C'est peut-être la signification de la densité relativement forte, au début de son œuvre, de narrateurs à l'agonie: deux des narrateurs de romans sont à la fin de leur vie 
(Non ora, non qui et Aceto, arcobaleno) et le narrateur de la nouvelle Il violino est même mort.

Chez De Luca, l'écriture est une patience, entre silence et violence, un piétinement lent et obstiné, où la progression de la diégèse procède souvent par retours, reprises, réitérations thématiques et lexicales sur lesquels en particulier Non ora, non qui, Aceto, arcobaleno et Tre cavalli sont entièrement construits. Elle ne coule pas d'un seul mouvement linéaire, mais rencontre des obstacles, des empêchements, des gênes figurées par les nombreuses infirmités distribuées aux personnages: surdité, cécité, claudication, bégaiement, mutisme. Corps et écriture portent en eux une suspension, un retard, une attente. Et c'est l'obstacle qui permet la trajectoire, comme dans un parcours d'escalade où, de prise en prise, contournant les difficultés et s'y appuyant, le grimpeur ouvre sa voie. Le défaut qui marque et discrimine devient une chance pour l'être, comme dans la Bible où Dieu ne traite pas différemment ses élus. Dans Una nuvola come tappeto, De Luca y insiste: Moïse bégaie, Jacob garde une claudication de son combat avec l'ange ( $N T$, p. 28 et 51 ).

En commun le traducteur athée de la Bible et le romancier moraliste ont une boussole, un nord de la vérité, un sommet où tendre. Dans l'univers de De Luca, les tâtonnements portent à la fulgurance. Mais la lumière à laquelle on touche est crue et dure, pétrie de violence, révélatrice de mort. Porteurs de différences qui les marquent, les êtres chez De Luca sont hors d'indifférence, et donc d'acceptation. Ils en brûlent. Les hommes âgés de Non ora, non qui et d'Aceto, arcobaleno, puis l'homme mûr de Tre cavalli ne sont qu'indirectement confrontés au choix du meurtre justicier, car d'autres, de plus en plus près d'eux, l'accomplissent. Plus tard dans l'œuvre, les adolescents narrateurs de Tu, mio et de Montedidio deviennent les acteurs directs du geste meurtrier vengeur. Non pas en vain, mais sans prise: il n'y a pas de place dans le fil de l'histoire des hommes pour un geste qui veut réparer l'offense. Ainsi rien n'est réglé de ce qui pourtant est consommé. Reste la responsabilité individuelle et historique, l'épaisseur de vie de chacun, de l'énergie pure pour agir sur le monde, maintenant, ici.

Chez De Luca, la lenteur de l'élocution ou de la démarche, la timidité, la maladresse et la pudeur, la capacité de silence accompagnent une force vitale d'enracinement au ras du sol, du sable, de la roche, de la rue. L'envol (le boomerang et l'ange de Montedidio) ou la plongée (la mer où nager, présente dans tous ses livres) sont le pendant utopique de la condition sociale qui lie chacun à une place, souvent tranchée de guerre ou galerie de terrassement, lieu de labeur et de danger ${ }^{25}$.

Rêve et limites chez De Luca cœxistent et se respectent. Et, toutes déréalisations manipulatrices battues en brèche, continuent de fabriquer de l'Histoire.

25. Parmi les nombreuses occurrences de la tranchée, voir tout particulièrement la nouvelle Una specie di trincea dans $A S$, p. 47-54. 


\section{DOMINIQUe VitToZ}

BIBLIOGRAPHIE d'Erri De Luca, par ordre chronologique

(toutes les traductions françaises sont dues à Danièle Valin)

\section{Écrits publiés en volume}

Non ora, non qui, Milan, Feltrinelli, 1989 (Une fois, un jour, Lagrasse, Verdier, 1992) : roman.

Lettere a Francesca, Naples, Alfredo Guida, 1990 : récit épistolaire.

Una nuvola come tappeto, Milan, Feltrinelli, 1991 (Un nuage comme tapis, Paris, Rivages, 1994): nouvelles.

Aceto arcobaleno, Milan, Feltrinelli, 1992 (Acide, arc-en-ciel, Paris, Rivages, 1994) : roman.

I colpi dei sensi, Rome, Fahrenheit 451, 1993 : nouvelles (intégrées dans le volume En haut à gauche, Paris, Rivages, 1996)

Prove di risposta, ed. Nuova Cultura, 1994; nouvelle édition revue et augmentée: Altre prove di risposta, Naples, Dante \& Descartes, 2000 : réflexions sur son travail d'écriture.

In alto a sinistra, Milan, Feltrinelli, 1994 (En haut à gauche, Paris, Rivages, 1996) : nouvelles.

Pianoterra, Macerata, Quodlibet di Tempi Provinciali, 1995 (Rez-de-chaussée, Paris, Rivages, 1996): recueil de chroniques publiées dans le quotidien Avvenire.

Alzaia, Milan, Feltrinelli, 1997 (Alzaia, Paris, Rivages, 1998): recueil de chroniques publiées dans le quotidien Avvenire.

Ora prima, Communauté de Bose, Qiqajon, 1997 (Première heure, Paris, Rivages, 2000): réflexions principalement inspirées par la Bible.

Tu, mio, Milan, Feltrinelli, 1998 (Tu, mio, Paris, Rivages, 1998) : roman.

La rivolta di Giobbe, en collaboration avec Y. Goll et G. Bertelli, Brescia, edizioni L'Obliquo, 1998.

Tre cavalli, Milan, Feltrinelli, 1999 (Trois chevaux, Paris, Gallimard, 2000) : roman.

Cattività. Ritratti dal carcere, photographies de Marco Delogu, Rome, Stampa alternativa, 1999 : réflexion sur la détention des ex-terroristes de gauche.

Un papavero all'occhiello senza coglierne il fiore, Montereale Valcellina, Circolo Menocchio, 2000 : recueil d'articles parus dans Il Manifesto entre 1998 et 1999.

Tufo, Naples, Libreria Dante \& Descartes, 2001: petit recueil de deux nouvelles (Tuf, in Meet, n. 4, 2001).

Montedidio, Milan, Feltrinelli, 2001 (Montedidio, Paris, Gallimard, 2002) : roman.

Opera sull'acqua, Torino, Einaudi, 2002 (Euvre sur l'eau, édition bilingue, Paris, Seghers, 2002): recueil de poésies.

Nocciolo d'oliva, Padoue, Edizioni Messaggero di Sant'Antonio, 2002 : vingt-six réflexions sur le nouveau et l'ancien Testament.

Lettere da una città bruciata, Naples, Libreria Dante \& Descartes, 2002 (deux de ces textes sont publiés en français Persichetti: ils ont besoin de toi. Lettre à un détenu politique flambant neuf et À la mémoire d'un étranger, in ouvrage collectif, Vingt ans après. Réfugiés italiens, vies en suspens, Paris, Nautilus, 2003, p.62-71): recueil de textes inédits ou publiés de façon éparse sur les années de la contestation et l'après terrorisme.

Il contrario di due, Milan, Feltrinelli, 2003 : recueil de nouvelles introduites par un poème sur la mère et sa disparition, dont certaines avaient déjà été publiées comme Vento in faccia (in MicroMega, 3, 2002, p. 255-260) et les cinq récits de I colpi dei sensi.

L'ultimo viaggio di Sindbad, Turin, Einaudi, 2003 (texte de théâtre sur le voyage en mer d'immigrés clandestins).

\section{Traductions commentées de la Bible}

Esodo/Nomi, Milan, Feltrinelli, 1994 : traduction du livre de l'Exode. Giona/Ionà, Milan, Feltrinelli 1995 : traduction du livre de Jonas. 
Kohèlet/Ecclesiaste, Milan, Feltrinelli, 1996 : traduction du livre de Qohélet.

L'urgenza della libertà. Il Giubileo e gli anni sacri nella loro stesura d'origine, Naples, Filema, 1999 : traduction et commentaire des douze premiers vers du chapitre vingt-cinq du Lévitique.

Libro di Rut, Milan, Feltrinelli 1999 : traduction du livre de Ruth.

Elogio del massimo timore. Il salmo secondo, Naples, Filema, 2000 : traduction du psaume deux et commentaire.

Vita di Sansone, Milan, Feltrinelli, 2002 : traduction des chapitres 13 à 16 du livre des Juges.

\section{Autres traductions}

«Naviga una preghiera nelle mie vene da anni» (traduction de poètes yiddish), in MicroMega, 3, 1997.

A. Kacyzne, Il fidanzamento a Partschew, in MicroMega, 1, 1999.

D. Katz, Nòah Hanshek dell'altro mondo, Naples, Libreria Dante \& Descartes, 2002.

\section{Textes épars (liste non exhaustive)}

"Giustizia fototessera», in Il Manifesto, 4 février 1990.

"Et lahavòk, la sera in cui tornarono gli abbracci», in Avvenire, 24 décembre 1994; maintenant in F. Pierangeli, Ultima narrativa italiana (1983-2000), Rome, Studium, 2000, p. $87-90$ (texte non repris dans Pianoterra).

"Terrorismo senza ragioni», in MicroMega, 5, 1995, p. 159-165 (dialogue entre Erri De Luca et Mimmo Calopresti à propos du film La seconda volta).

"Una storia di strada», in MicroMega, 5, 1995, p. 166-168 (une version remaniée et raccourcie figure maintenant dans le recueil Lettere da una città bruciata).

"Lettere dai tempi supplementari», in MicroMega, 4, 1996, p. 203-216 (échange épistolaire entre Erri De Luca et Ovidio Bompressi en marge du procès Calabresi daté janvier-mai 1990).

«La scrittura e la storia. A proposito di Izet Sarajlic, poeta di Bosnia, testimone di una guerra contro Dio, contro il Dio dell'altro. Con due poesie inedite e una lettera", in MicroMega, 5, 1996, p. 190-194.

Lettera non chiusa, in Arnaldo Colasanti (sous la direction de), Decalogo. Dieci scrittori raccontano $i$ dieci comandamenti, Milan, Rizzoli, 1997 (De Luca traite le commandement «Tu ne désireras pas la femme d'autrui»; autres textes d'Aurelio Picca, Luca Doninelli, Linda Ferri, Carola Susani, Rocco Carbone, Laura Pariani).

"Esiste ancora il lieto fine?», in MicroMega, 1, 1997, p. 188-191 (échange de lettres entre Erri De Luca et Izet Sarajlic).

«I tamburi di Napoli spazzano via il Sud piagnone», in Corriere della Sera, 27 mars 1998.

"Breve risposta a un questionario lungo ", in MicroMega, 2, 1999, p. 87-88 (dix définitions acerbes du gouvernement de la gauche en Italie).

"Risposta a Sofri. Non esistono attentatori», in Il Manifesto, 3 juin 1999 (texte non inclus dans Un papavero all'occhiello senza coglierne il fiore).

Préface et Le radical "commun", in P. Persichetti et O. Scalzone, La révolution et l'État. Insurrections et "contre insurrection" dans l'Italie de l'après-68: la démocratie pénale, l'état d'urgence, Paris, Dagorno, 2000, p. 9-12 et p. 323-324.

Introduzione, in D. De Marco, La mia ala è pronta al volo, Spilimbergo, Edizioni Interattiva, 2001.

Mostar, in ouvrage collectif, Carnets de Sarajevo. Rencontres européennes du livre, $\mathrm{n}^{\circ} 1$, Paris, Gallimard, 2002, p. 45-46.

Prefazione, in L. Viola, Pellegrino a pedali. Viaggio a Santiago di un nomo di poca fede, Cuneo, Primalpe, 2002. 


\section{DOMINIQUe VitToz}

A Catherine Izzo, in Noirs silences. Dans le sillage des "Marins perdus", photographies de Catherine

Izzo, textes d'Erri de Luca et Thierry Fabre, Marseille, Images en Manœuvre, 2002, p. 8-9.

"I campi dell'infamia", in Il Manifesto, 29 novembre 2002.

"Quando ti piglieranno digli che sei figlio di Maria», in Corriere. it, 22 décembre 2002.

"Une illégalité affichée», traduit par Guillaume Chpaltine, in Les Inrockuptibles, janvier 2002, p. 32-33.

"L'islam est notre frère de sang", in Télérama, 29 janvier 2003, p. 18-19.

Prefazione, in A. Ballerini e A. Benna, Il muro invisibile, Fratelli Frilli editori, 2003. 